\title{
Philosophiques
}

\section{Jean-Pierre Cometti, La maison de Wittgenstein , Paris, PUF (coll. "Perspectives critiques »), 1998, 254 p.}

\section{Pasquier Lambert}

Volume 26, numéro 2, automne 1999

La critique de la raison en Europe centrale

URI : https://id.erudit.org/iderudit/004942ar

DOI : https://doi.org/10.7202/004942ar

Aller au sommaire du numéro

Éditeur(s)

Société de philosophie du Québec

ISSN

0316-2923 (imprimé)

1492-1391 (numérique)

Découvrir la revue

Citer ce compte rendu

Lambert, P. (1999). Compte rendu de [Jean-Pierre Cometti, La maison de Wittgenstein, Paris, PUF (coll. « Perspectives critiques »), 1998, 254 p.]

Philosophiques, 26(2), 388-391. https://doi.org/10.7202/004942ar d'utilisation que vous pouvez consulter en ligne.

https://apropos.erudit.org/fr/usagers/politique-dutilisation/ 


\section{Comptes rendus}

Jean-Pierre Cometti, La maison de Wittgenstein. Paris, PUF (coll. «Perspectives critiques »), 1998, 254 p.

Ce livre est divisé en neuf courts chapitres se présentant sous la forme d'un essai continu plutôt qu'ayant la structure d'une étude théorique. O n retrouve, dans ce deuxième recueil consacré à Wittgenstein par I'auteur après Philosopher avec Wittgenstein (PUF, 1996), un ensemble d'articles refondus en vue d'illustrer « les voies de l'ordinaire » (sous-titre du recueil) suivies par Wittgenstein, qui fut aussi bien ingénieur et architecte que jardinier, instituteur et musicien, comme si la philosophie était une activité trop abstraite avec laquelle il devait rompre sans pourtant jamais réussir à y échapper : «Le philosophe est quelqu'un qui doit guérir en lui-même de nombreuses maladies de l'entendement avant de pouvoir parvenir aux saines notions du sens commun », admet W ittgenstein. Croyant en avoir terminéavec la philosophieen 1920 après la rédaction du Tractatus, Wittgenstein changea souvent d'occupation, peut-être davantage par embarras moral que par désir. II reprit la philosophie vers 1929 et enseigna à Cambridge jusqu'en 1947, mais vécut toujours la pratique de la philosophie avec l'ombre de la mauvaise conscience planant sur lui.

II faut entendre le titre de ce livre en un sens littéral aussi bien que figuré, car si la philoso phie de W ittgenstein s'offre au lecteur comme un foyer de réflexions habitable, la maison dont il fit les plans avec Paul Engel mann, puis dirigea la construction et la finition pour sa sœur $\mathrm{G}$ retl ( $M$ argarete Stonborough) en 1928, représente un geste concret de la part de Wittgenstein. Ce geste est loin d'être le seul de ce genre puisqu'il avait déjà construit une machine à coudre à l'âge d'environ dix ans et dessiné les plans d'un turboréacteur lors de ses études à I'U niversité de $M$ anchester vers 1910. Ces plans sont d'ailleurs actuellement déposés à la bibliothèque del'Université. L'itinéraire de Wittgenstein est donc un peu surprenant, car « les philosophes sont plus volontiers constructeurs de systèmes que de maisons » (p. 14), constate Cometti dans le premier chapitre « Le geste de l'architecte».

La construction de Wittgenstein évoque la rigueur et la concision du Tractatus, mais l'auteur refuse les comparaisons, parfois excessives il est vrai, entrel'œuvre philosophique de Wittgenstein et ses réalisations techniques et artistiques. II invoque à cet égard l'autorité de Karl Kraus, car l'œuvre de W ittgenstein participe en effet bien souvent du même l'esprit : « Tout ce que nous partageons, A. Loos [architecte viennois ayant influencé Engelmann et Wittgenstein] et moi - lui matériellement et moi verbalement - a toujours consisté à dire qu'il existe une différence entre une urne et un pot de chambre. $M$ ais chez les hommes d'aujourd'hui, il y a ceux qui se servent du pot de chambre comme d'une urne et ceux qui utilisent l'urne comme un pot de chambre » (cité par l'auteur, p. 19). À l'époque, tout à Vienneétait décoré, avec excès et parfois le goût le plus mauvais qui soit, des poignées de porte aux pichets de bière. La critique de l'ornementalisme viennois est de ce fait très présente dans la maison de style minimaliste de Wittgenstein, qui s'oppose par sa froideur et son dépouillement à l'utilisation de fioritures et de garnitures inutiles, rappelant les idées de Loos sur le design des objets ou des habitations, qui devait être envisagé uniquement d'après la fonctionnalité. L'auteur complète ce chapitre par de judicieux commentaires sur les 


\section{4 · Philosophiques / Automne 1999}

remarques de W ittgenstein concernant I'architecture, I'esthétique, la fonction de la philosophie et de l'art. Une remarque telle que : "Éthique et esthétique sont une », par exemple, vient souligner la valeur de manifeste éthique de toute réalisation artistique. Et lorsque W ittgenstein affirme : « Le travail en philosophie - comme, à beaucoup d'égards, le travail en architecture - est avant tout un travail sur soi-même. C'est travailler à une conception propre. À la façon dont on voit les choses », il insiste encore plus explicitement sur les liens entre la vocation éthique et thérapeutique qu'il assigne à la philosophie (ou à son dépassement) et celle de l'architecture, qui est d'exprimer la pureté de l'éthique et la perfection à laquelle une thérapeutique doit nous faire parvenir. Wittgenstein aimait d'autre part à répéter que « dans la vie, il ne faut s'encombrer de rien », exprimant par là à la fois une éthique et une esthétique du dénuement, ainsi qu'une revalorisation del'« ordinaire».

Le deuxième chapitre, «Wittgenstein secret », est hautement intéressant, du fait qu'on y retrouve de nombreuses citations des Carnets secrets, qui ne sont pas encore traduits en français puisque la seule version est une édition non autorisée en italien. L'auteur fait état, en étayant son propos de citations de ces carnets, de l'épreuve très difficile que fut la guerre pour Wittgenstein. Ce dernier consigne ses commentaires à propos des membres de l'équipage du bateau sur lequel il était embarqué durant la guerre et exprime une grande détresse morale et spirituelle. Pour tout dire, il les juge odieux et insupportables. Profondément préoccupé par des problèmes de logique auxquels vont suivre bientôt des problèmes éthiques qui aboutissent à la rédaction du Tractatus, il se sent totalement étranger face à ces hommes vulgaires qui n'ont aucun respect pour lui ni pour leurs autres compagnons. L'auteur montre que c'est dans l'A brégé des É vangiles de Tolstoï que Wittgenstein aurait trouvé où puiser un support psychologique et spirituel, et que certaines idées contenues dans cet ouvrage auraient du coup été assimilées par Wittgenstein pour se retrouver dans sa conception de l'éthique et de la vie « bonne » ou " correcte». Cette expérience a évidemment largement influencé l'attitude et la façon de penser de Wittgenstein : elle a même eu des répercussions importantes sur les idées développées dans le Tractatus, notamment sur celles qui concernent l'éthique, le rel igieux et le mystique. Cela suffit à montrer que les faits appartenant à la vie personnelle de Wittgenstein ont une incidence sur sa pensée et qu'il est inutile d'en nier la pertinence.

W ittgenstein passait un jour devant une librairie de Cambridge et aperçut les portraits de Russell, Freud et Einstein. Un peu plus loin, il vit dans un magasin de musique ceux de Beethoven, Schubert et Chopin, et, comparant ces portraits avec les précédents, il ressentit intensément la terrible dégénérescence qui s'était abattue sur I'esprit humain en aussi peu qu'une centaine d'années. Cette anecdote rapportée par Drury, un ami de W ittgenstein, est citée par l'auteur dans son troisième chapitre intitulé « Le portrait d'Einstein ». Il vise dans ces pages à cerner le rapport que W ittgenstein avait avec l'esprit de son temps afin de comprendre son statut de contemporain inactuel, farouchement opposé aux tendances qui se développent et sont défendues à l'époque. A près I'injonction au silence du Tractatus, W ittgenstein, incapable de parvenir malgré tout à « la paix dans les pensées » à laquelle aspire le philosophe, cherche à « s'engager dans une activité dont la valeur s'éprouve à l'apaisement qu'elle est susceptible d'offrir » (p. 84). C'est pourquoi le nouvel esprit qui l'anime est profondément différent de celui de la civilisation américaine et européenne, puisque son but n'est pas d'édifier de nouvelles constructions philosophiques, mais d'avoir clairement 
sous les yeux les fondements de ces constructions, comme il l'affirme dans plusieurs projets de préface.

Dans « $M$ irages de la modernité », le quatrième chapitre, l'auteur montre les affinités de Wittgenstein avec l'esprit viennois, malgré son conservatisme et le fait qu'il ait finalement peu habité $V$ ienne. $Z$ w eig et $H$ ofmannsthal ont étéles témoins des métamorphoses uniques de Vienne au début du siècle : Schönberg, Berg et Webern remplacent en musique Brahms et Bruckner, tandis qu'une révolution s'opère en architecture avec Loos ; Freud développe la psychanalyse, Broch et M usil entreprennent d'immenses projets en littérature. Ce monde est en partie celui de W ittgenstein, même si celui-ci est resté attaché à un autre âge : il préfère $G$ œthe et $M$ örike, Brahms, Beethoven et Schubert aux écrivains et musiciens de son temps, et deviendra un lecteur occasionnel, mais très critique, de Freud. L'auteur évoque ensuite certains rapprochements entre W ittgenstein et Trakl et tente de cerner la position de W ittgenstein à l'égard de la poésie et de l'indicible. Cet aspect intéressant et peu traité dans la littérature wittgensteinienne aurait cependant mérité de plus amples développements.

Dans le cinquième chapitre, « Les faubourgs du langage: Wittgenstein et $H$ eidegger », l'auteur se lance dans une comparaison entre les conceptions wittgensteinienne et heideggerienne du langage. Ce projet ambitieux a déjà été tenté par certains, mais ces deux philosophes qu'on semble reconnaître comme étant, après H usserl, les deux plus influents de ce siècle, peuvent difficilement faire l'objet d'un traitement commun, sauf pour montrer qu'ils n'ont précisément pas grand-chose en commun.

Le sixième chapitre, «A pologie de l'ordinaire », revient sur le thème principal de l'ouvrage en partant de l'esthétique minimaliste de W ittgenstein, une esthétique qui valorise une approche « ordinaire » de la compréhension de l'œuvre d'art. W ittgenstein pense que ce qui sert l'œuvre d'art ne relève pas du langage, par exemple des adjectifs qu'on peut utiliser pour exprimer ce qu'on ressent en face d'une œuvre, mais plutôt du geste, qui sert à indiquer une compréhension de l'œuvre, comme lorsqu'on indique qu'un poème doit être lu en ponctuant autrement ou qu'une pièce doit être jouée avec un tempo différent. Le septième chapitre intitulé «Éloge dela lenteur » est l'occasion pour l'auteur de s'arrêter sur le style de Wittgenstein et de préciser justement le rôle que joue le tempo dans la lecture et le processus de compréhension du langage. Comprendre, ce serait être capable de donner une opinion, d'adopter un point de vue, et non pas simplement dire qu'une œuvre est belle ou charmante, ce que n'importe qui peut faire, mais pouvoir montrer qu'elle est juste ou correcte dans tel ou tel de ses aspects, ce qui démontre une réelle compréhension.

L'auteur poursuit dans le chapitre huit, «L'attrait de l'imprévisible », son investigation sur la fonction essentielle de l'esthétique dans la pensée de Wittgenstein, ainsi que la part du geste et l'imprévisibilité des applications des règles dans les divers « jeux de l'art » et « jeux de langage sur l'art » (p. 201). Dans le dernier chapitre enfin, "L'esprit des mots », l'auteur essaie de cerner ce que représente chez Wittgenstein l'expérience de la signification et en particulier le rôle du langage dans l'expression de l'intériorité. On sait que Wittgenstein s'attaque en effet à tous les mythes de l'intériorité et des corps de signification, cherchant à dissoudre toute forme d'essentialisme au profit d'un usage ordinaire du langage vérifiable par des critères publics.

Q uelques éléments de biographie sont donnés en appendice dans l'espace d'une dizaine de pages, suivis d'une abondante bibliographie et d'un index des noms. 
Le livre de Cometti, pour autant qu'il soit lu comme un essai, démontre une connaissance approfondie de l'œuvre wittgensteinienne par ses analyses pénétrantes des rapports entre la vie et I'œuvre de W ittgenstein, ce dernier ayant réhabilitéle langage ordinaire et la vie quotidienne contre les tendances de la tradition, marchant sur « les voies de l'ordinaire » que méprisent généralement les philosophes. L'immense effort de W ittgenstein aura peut-être été, dans I'optique d'une thérapie du soi par soi, de rechercher constamment la chair concrète du monde - l'« ordinaire » luimême - afin de ne pas sombrer dans les trop purs délices de la folie : « J e crains souvent la folie ». "Dans la santé de l'entendement, nous sommes environnés par la folie ${ }^{1}$.

PASQUIER LAMBERT

Université de Paris I

1. Citations tirées des Vermischte B emerkungen, comme toutes les citations précédentes de Wittgenstein, sauf «Éthique et esthétique sont une », qui provient du Tractatus. 